\title{
A hipertensão arterial e o exercício físico: elementos para uma prescrição médica
}

José Pedro Marques, ${ }^{1}$ João Páscoa Pinheiro, ${ }^{2}$ Manuel Teixeira Veríssimo, ${ }^{3}$ Domingos Ramos ${ }^{4}$

\section{RESUMO}

O aumento dos níveis de atividade física está associado a benefícios globais na saúde do indivíduo e meta-análises recentes mostram que o exercício físico pode ter uma eficácia na redução da pressão arterial comparável à terapêutica anti-hipertensora de primeira linha.

Tradicionalmente considerava-se que o efeito anti-hipertensor do exercício físico era exclusivo do exercício de endurance e que o exercício de resistência, nomeadamente na sua vertente isométrica, seria prejudicial. Trabalhos publicados nos últimos anos parecem contrariar esta suposição. A evidência disponível é escassa mas aponta para que possa vir a desempenhar um papel na prevenção primária da hipertensão. Está também demonstrada a sua capacidade de influenciar positivamente outros fatores de risco cardiovascular como são a hipertrigliceridémia e o excesso de peso.

O exercício físico assume um papel na prevenção primária e secundária da hipertensão. Na generalidade dos doentes, os benefícios associados à sua prática ultrapassam os potenciais riscos. Nos indivíduos em que estes riscos não sejam desprezáveis deve haver lugar a uma avaliação médica que permita a prescrição de um programa de exercício seguro e orientado para o paciente.

Palavras-chave: Hipertensão Arterial; Exercício Físico; Atividade Física.

\section{INTRODUÇÃO}

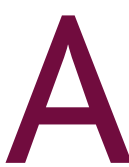
hipertensão arterial (HTA) é definida pela elevação crónica da pressão arterial sistólica (PAS) acima de 140mmHg e da pressão arterial diastólica (PAD) acima de 90mmHg. A prevalência de HTA é de $42 \%^{1}$ em Portugal e de $31 \%$ nos Estados Unidos da América. ${ }^{2}$ É um dos principais fatores de risco cardiovascular $^{3}$ contribuindo de forma significativa para a morbilidade e mortalidade por enfarte agudo do miocárdio e acidente vascular cerebral. Ao longo das últimas décadas, a evidência aponta para o aumento de uma relação doseresposta in-versa entre o volume total de exercício físico (EF) e a mortalidade cardiovascular. Neste artigo abordamos a eficácia anti-hipertensora do EF, comparando-a com a dos fármacos mais habitualmente empregues para esse

${ }^{1}$ Médico Interno de Medicina Desportiva; Centro Hospitalar e Universitário de Coimbra.

${ }^{2}$ Professor de Medicina, Médico Assistente Hospitalar Graduado de Medicina Física e Reabilitação, Especialista em Medicina Desportiva; Faculdade de Medicina da Universidade de Coimbra, Centro Hospitalar e Universitário de Coimbra.

${ }^{3}$ Professor de Medicina, Médico Assistente Hospitalar Graduado de Medicina Interna, Especialista em Medicina Desportiva; Faculdade de Medicina da Universidade de Coimbra, Centro Hospitalar e Universitário de Coimbra.

${ }^{4}$ Médico Assistente Graduado de Cardiologia, Mestre em Medicina Desportiva; Centro Hospitalar e Universitário de Coimbra. fim. Revemos o impacto de diferentes tipos de exercício e propomos um programa de exercício adaptado a esta população. Para melhor compreensão optou-se por definir no Quadro I alguns dos conceitos usados nas secções subsequentes.

\section{EFICÁCIA ANTI-HIPERTENSORA DO EF}

É atualmente consensual que perante um doente com hipertensão o médico deve recomendar alterações ao seu estilo de vida ${ }^{4}$ e zelar para que, tanto quanto possível, elas sejam cumpridas. Estas alterações passam forçosamente pelo aumento da atividade física. Existe inclusive uma recomendação (classe I, nível B) segundo a qual, no género feminino, 150 minutos de atividade física semanal de intensidade moderada deve ser usada. ${ }^{5}$ Com base nesta recomendação somos levados a pensar que a medicação anti-hipertensora tem uma eficácia e/ou potência bastante superior à do EF. Um trabalho publicado em 2000 reunia os vários ensaios que tinham testado a eficácia de diferentes classes de anti-hipertensores e também aqueles que comparavam estratégias mais e menos intensivas. ${ }^{6}$ Podemos ver no Quadro II os resultados obtidos nalguns destes estudos.

É deveras interessante comparar estes resultados com 
QUADRO I. Conceitos-chave

Atividade física: corresponde a qualquer movimento do corpo humano que aumente o dispêndio energético.

Exercício físico: atividade física programada, estruturada e repetitiva para melhorar ou manter um ou mais componentes da aptidão física.

1-RM: quantidade máxima de peso que pode ser movimentado com a técnica adequada em apenas uma repetição de um exercício específico. $O$ teste para determinar o 1-RM pode ser utilizado como um limite superior, a fim de determinar a carga desejada para se realizar um exercício (como uma percentagem de 1RM).
Exercício de endurance/aeróbio: envolve grandes grupos musculares em atividades dinâmicas repetitivas que resultam em aumento da frequência cardíaca e dispêndio de energia.

Exercício de resistência: realizado contra uma força específica que se lhe opõe e que tem como objetivo aumentar a força e/ou resistência muscular. Pode ser dinâmico, quando a contração muscular se associa a alterações no comprimento e tensão do músculo e isométrico, quando da contração muscular resulta alteração no comprimento do grupo muscular envolvido.

MET: medida fisiológica que expressa o custo energético das atividades físicas. 1-MET equivale à energia suficiente para um indivíduo se manter em repouso. Quando se exprime o gasto de energia em METs representa-se o número de vezes pelo qual o metabolismo de repouso foi multiplicado durante uma atividade. os apresentados na meta-análise de Cornelissen et al, ${ }^{7}$ resumidos no Quadro III.

Comparações diretas são limitadas pelas diferentes metodologias usadas. No entanto, é notória a semelhança no que diz respeito à magnitude dos efeitos atingidos. No caso do exercício de endurance, se atentarmos apenas aos resultados obtidos na população de doentes com hipertensão, observaram-se reduções de pressão arterial (PA) de 8.3/5.2 mmHg. Estes são, na pior das hipóteses, comparáveis aos resultados obtidos com a terapêutica anti-hipertensora de primeira linha. Acresce o facto do EF ter efeitos pleiotrópicos, atuando sobre a obesidade, dislipidémia e insulinorresistência. Isto ajuda a explicar o benefício global no perfil cardiovascular, independentemente das reduções na PA. Apesar disso, diariamente constatamos que no nosso país a terapêutica não farmacológica (na qual se inclui a atividade física) da hipertensão permanece num segundo plano.

\section{COMPARAÇÃO ENTRE DIFERENTES TIPOS DE EF}

Tradicionalmente considerava-se que o efeito anti-hipertensor do EF era um exclusivo do exercício de endurance. Ao exercício de resistência, nas suas vertentes dinâmica e isométrica, era atribuída uma resposta hipertensora, o que contribuía para que este fosse contra-indicado na prevenção primária e secundária da hipertensão. Uma meta-análise publicada recentemente examinou o efeito de diferentes tipos de exercício na pressão arterial em repouso. ${ }^{7}$ Incluiu ensaios clínicos randomizados, com duração superior a quatro semanas, e em que participa-

$\begin{aligned} & \text { QUADRO II. Efeito anti-hipertensor das várias classes de fármacos em uso comum: } \\
& \text { resultados da análise prospectiva de ensaios clínicos randomizados (adaptado) }\end{aligned}$
\begin{tabular}{l|l|c|c|} 
Estudo & Descrição sumária & Diferenças na PA (tratamento - controlo) \\
\cline { 2 - 4 } & & PAS (mmHg) & PAD (mmHg) \\
\hline Hope & Ramipril vs placebo & -3 & -1 \\
SCAT & Enalapril vs placebo & -4 & -3 \\
PREVENT & Amlodipina vs placebo & -5 & -4 \\
ABCD - hipertensive & Enalapril vs Nisoldipina & $<1$ & $<1$ \\
HOT & Grupo tratamento intensivo & -3 & -3 \\
& (PAD alvo <80) vs controle & & \\
UKPDS-HDS & (PAD alvo >80) & +1 & +2 \\
\hline
\end{tabular}




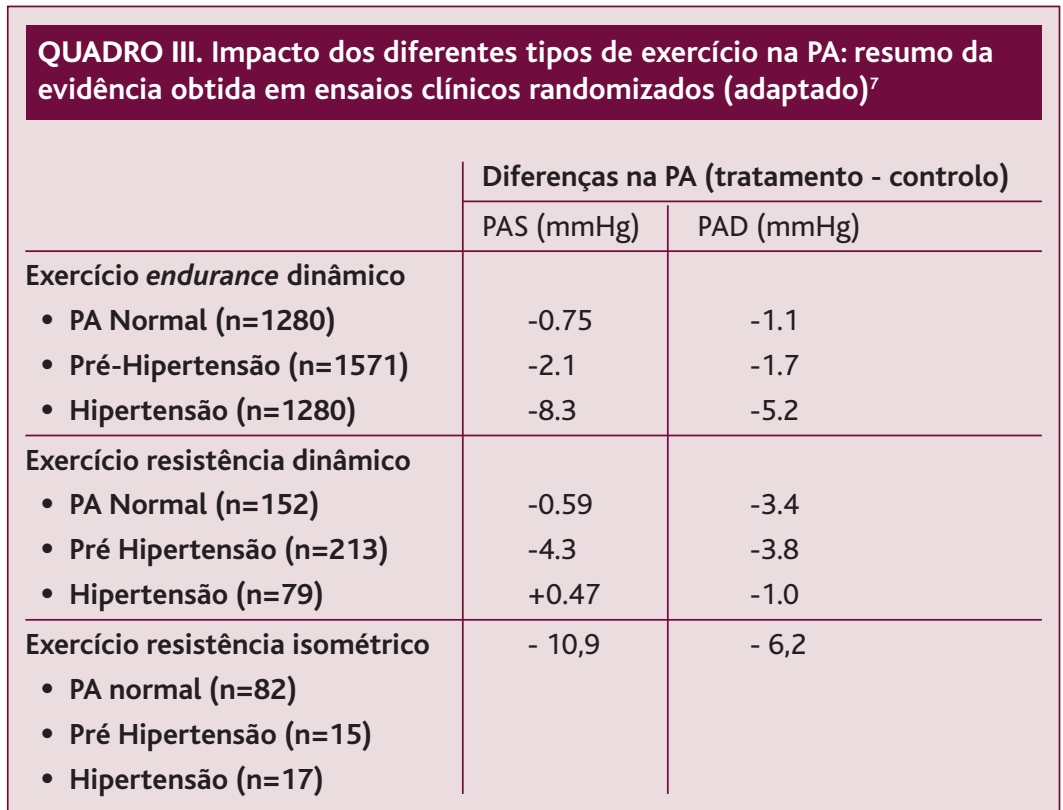

$\mathrm{n}=$ número total de indivíduos incluídos na meta-análise por categoria de PA. Não inclui indivíduos que participaram em trabalhos com diferentes braços de exercício (ver suplemento S2). ${ }^{7}$

promissores, mas provêm de um número reduzido de pacientes envolvidos $(\mathrm{n}=114),{ }^{7}$ na sua maioria normotensos e pré-hipertensos. Aguardamos com expectativa futuros trabalhos que testem os seus efeitos em populações maiores e nas diferentes fases do continuum que é a doença hipertensiva.

À luz do conhecimento atual parece sensato incluir o exercício de resistência nos programas de prevenção primária (e porventura secundária) da hipertensão. A sua eficácia na redução da PA em pessoas com hipertensão ainda carece de confirmação, mas a capacidade de influenciar favoravelmente a capacidade aeróbia, perfil lipídico e gordura (adiposidade) corporal tornam--no um complemento importante. ${ }^{8}$

\section{PRESCRIÇÃO DE EF NO DOENTE HIPERTENSO}

Apesar dos reconhecidos benefícios no

ram adultos com PA que vão desde valores normais até à hipertensão. Foi demonstrada a redução significativa da PAS e PAD com os exercícios de endurance, de resistência dinâmica e resistência isométrica (ver Quadro III). Através da análise de subgrupos, os autores chegaram a conclusões interessantes no que diz respeito ao exercício de endurance: é maior a eficácia nos indivíduos hipertensos (-8.3/ -5.2) quando comparado com pré-hipertensos $(-2,1 /-1,7)$ e normotensos $(-0,75 /-1,1)$; os programas com duração inferior a 24 semanas parecem reduzir mais a PAS $(p<0,0001)$ e a PAD $(p<0,01)$ do que aqueles com duração superior; sessões de menos de 210 minutos por semana estão associadas a reduções significativamente maiores da PAS $(p<0,05)$ e não tão significativas da PAD $(p=0,20)$; sessões de menor intensidade estão associadas a efeitos menos significativos. Estes achados estarão provavelmente relacionados com o facto de programas de duração superior estarem associados a diminuição da compliance e de sessões de exercício com maior duração implicarem habitualmente uma diminuição da intensidade. O efeito hipotensor do exercício de resistência dinâmica parece ser maior em populações normotensas e pré-hipertensas. A verdade é que, até à data e à semelhança do que acontece com o exercício isométrico, poucos estudos avaliaram os seus efeitos em doentes com hipertensão. Os resultados obtidos com o treino de resistência isométrico são bastante tratamento da hipertensão, o EF não é isento de riscos. Como tal, alguns indíviduos carecem de avaliação médica antes de iniciarem a atividade física. Os principais objetivos desta avaliação são:

- Identificar contra-indicações absolutas ou relativas à prática de determinado tipo de atividade física/desporto.

- Adaptar, tanto quanto possível, o programa de exercício à condição clínica e preferência do indivíduo, de forma a minimizar as complicações e promover a compliance.

- Identificar doença cardiovascular (DCV) oculta.

- Identificar indivíduos com risco cardiovascular elevado, como aqueles com hipertensão não controlada, angina instável e diabetes descompensada. Estes devem ser estabilizados antes de iniciar o programa de exercício.

Obviamente a profundidade da avaliação pré-participação vai depender da intensidade planeada para o programa de exercício e do perfil de risco cardiovascular. ${ }^{9} \mathrm{O}$ Quadro IV apresenta as indicações para realização de prova de esforço. Um paciente assintomático incluído no grupo de risco $\mathrm{A}$ ou $\mathrm{B}$, com $\mathrm{PA}<180 / 110 \mathrm{mmHg}$ e que pretenda participar em exercício de intensidade ligeira a moderada ( $<60 \% \mathrm{VO}_{2}$ max) não necessita habitualmente de investigação diagnóstica adicional. Já os incluídos no grupo C, 
sem DCV ou TA $>180 / 110 \mathrm{mmHg}$, podem beneficiar de prova de esforço caso pretendam exercitar-se com intensidade moderada ( 40 a $60 \% \mathrm{VO}_{2}$ max). A prova de esforço é indispensável para todos os pacientes com DCV documentada, independentemente do nível de intensidade pretendido. Para além disso, o exercício vigoroso nestes pacientes deve ser restrito a centros de reabilitação cardíaca. ${ }^{10-11}$

No que concerne à terapêutica farmacológica existem alguns cuidados a ter no doente ativo com hipertensão. ${ }^{10-}$ ${ }^{11}$ Os fármacos escolhidos devem baixar a PA em repouso e durante o exercício, as resistências vasculares periféricas e preferencialmente não afetar a capacidade máxima de exercício. Por estas razões preferem-se os inibidores da enzima de conversão da angiotensina, antagonistas dos recetores da angiotensina e bloqueadores de canais de cálcio. Alguns aspetos particulares das classes farmacológicas habitualmente usadas devem ser tidos em conta: os bloqueadores de canais de cálcio, os vasodilatadores diretos e os bloqueadores alfa podem provocar uma resposta hipotensora (no pós-exercício imediato) exagerada, devendo aconselhar-se o prolongamento do período de arrefecimento; a utilização de tiazidas deve ser ponderada com cautela devido ao risco de hiper e hipocalcemia, hiponatremia, hipocaliemia e potenciais arritmias malignas; diuréticos e bloqueadores-beta podem afetar a termorregulação e aumentam o risco de hipoglicémias; os diuréticos de ansa podem causar depleção de volume; os bloqueadores-beta têm efeitos inotrópicos e cronotrópicos negativos. Os não seletivos podem provocar broncoconstrição e em doentes com doença arterial periférica podem agravar a claudicação intermitente. A utilização dos bloqueadores-beta em hipertensos ativos fisicamente deve ser restrita a casos excecionais.

O programa ideal para obter a redução da TA em doentes hipertensos ainda não foi estabelecido. No entanto, de acordo com a evidência mais recente e recorrendo ao FITT, ${ }^{9}$ propõe-se o seguinte:

- Frequência: três a cinco vezes por semana para o exercício aeróbio.

- Intensidade: moderada $\left(40 \%<\mathrm{VO}_{2} \mathrm{R}<60 \%\right.$ ou $12-13$ na escala de Borg). Em pacientes selecionados podem ser obtidos benefícios adicionais com intensidades superiores. ${ }^{9,11}$

- Tempo: 30 a 60 minutos por sessão de exercício continuado ou intermitente (mínimo de 10 minutos de cada vez).

- Tipo: O mais estudado é o exercício aeróbio, dentro do qual se podem recomendar as caminhadas, jogging, ciclismo, natação. A preferência individual deve ser tida em conta para maximizar a aderência.

O exercício de resistência integra a generalidade dos programas de prescrição de exercício na população geral e em subpopulações de pacientes com patologias diversas. ${ }^{11}$ No doente com hipertensão poderá ser recomendado em associação ao exercício aeróbio, a realizar duas a três vezes por semana, preferencialmente com intensidade $>60 \%$ de 1-RM e envolvendo os principais grupos musculares (cinturas escapular e pélvica, musculatura abdominal e dorsal, flexores e extensores dos membros superior e inferior).

\section{CONCLUSÃO}

O aumento da atividade física é condição sine qua non para a adoção de um estilo de vida mais saudável. A capacidade do EF influenciar favoravelmente o perfil tensional ficou bem patente neste texto. Enquanto a evidência em relação ao exercício de endurance é já robusta, o mesmo não se passa com o exercício de resistência. São necessários mais trabalhos que permitam caracterizar a resposta da PA ao exercício de resistência em pacientes hiperten-

\section{QUADRO IV. Algoritmo de decisão para a necessidade de prova de esforço no âmbito da avaliação pré-participação (adaptado) ${ }^{9}$}

\begin{tabular}{l|l|l|l|}
$\begin{array}{l}\text { Pressão arterial } \\
(\mathrm{mmHg})\end{array}$ & $\begin{array}{l}\text { Grupo A (sem fatores de risco; } \\
\text { sem DOA/DCV) }\end{array}$ & $\begin{array}{l}\text { Grupo B (pelo menos 1 fator de } \\
\text { risco não incluindo diabetes; sem } \\
\text { DOA/DCV) }\end{array}$ & $\begin{array}{l}\text { Grupo C (DOA/CVD e/ou } \\
\text { diabetes, com ou sem outros } \\
\text { fatores de risco) }\end{array}$ \\
\hline $130-139 / 85-89$ & Vigoroso & Vigoroso & Moderado ou Vigoroso \\
$140-159 / 90-99$ & Vigoroso & Vigoroso & Moderado ou Vigoroso \\
$>160 />100$ & Controlar a TA em primeiro lugar & Controlar a TA em primeiro lugar & Controlar a TA em primeiro lugar \\
\hline
\end{tabular}

DOA: doença de órgão-alvo; DCV: doença cardiovascular

Exercício moderado: 40 a 60\% VO2 max - Exercício vigoroso: > 60\% VO2 max 
sos. Importa destacar que grande parte dos dados que apresentamos é extraída da meta-análise de Cornelissen et $\mathrm{al}^{7} \mathrm{e}$, portanto, a sua interpretação é condicionada pelas limitações da técnica meta-analítica e da literatura primária incluída. À primeira vista podem parecer resultados modestos, mas quando enquadrados na perspetiva de que a redução de $5 \mathrm{mmHg}$ na PAS é responsável por uma diminuição de $14 \%$ na mortalidade por AVC e $9 \%$ na mortalidade por doença coronária, ${ }^{4}$ certamente já não fica essa impressão. Num trabalho que incluiu mais de 100.000 participantes, Kodama et al demonstraram que o aumento de apenas 1 MET na capacidade aeróbia está associado a reduções de, respetivamente, 13 e 15\% na mortalidade global e por doença cardiovascular. ${ }^{12}$ Apesar disso, a adesão da população ao EF permanece reduzida. É imperioso apurar os motivos subjacentes a esta realidade e incentivar a comunidade médica a prescrever EF com base na atual evidência clínica.

\section{REFERÊNCIAS BIBLIOGRÁFICAS}

1. Macedo ME, Lima MJ, Silva AO, Alcântara P, Ramalhinho V, Carmona J. Prevalência, conhecimento, tratamento e controlo da hipertensão em Portugal: estudo PAP [Prevalence, awareness, treatment and control of hypertension in Portugal: the PAP study]. Rev Port Cardiol. 2007;26(1):21-39. Portuguese

2. Centers for Disease Control and Prevention. Vital signs: prevalence, treatment, and control of hypertension: United States, 1999-2002 and 20052008. MMWR. 2011;60(4):103-8.

3. World Health Organization. The world health report 2002: reducing risks, promoting healthy life. Geneva:WHO; 2002.

4. Chobanian AV, Bakris GL, Black HR, Cushman WC, Green LA, Izzo JL Jr, et al. The seventh report of the Joint National Committee on Prevention, Detection, Evaluation, and Treatment of High Blood Pressure: the JNC 7 re- port. JAMA. 2003;289(19):2560-72.

5. Mosca L, Benjamin EJ, Berra K, Benzanson JL, Dolor RJ, Lloyd-Jones DM, et al. Effectiveness-based guidelines for the prevention of cardiovascular disease in women - 2011 update: a guideline from the American Heart Association. J Am Coll Cardiol. 2011;57(12):1404-23.

6. Neal B, MacMahon S, Chapman N, Blood Pressure Lowering Treatment Trialists' Collaboration. Effects of ACE inhibitors, calcium antagonists, and other blood-pressure-lowering drugs: results of prospectively designed overviews of randomised trials. Lancet. 2000;356(9246):1955-64.

7. Cornelissen VA, Smart NA. Exercise training for blood pressure: a systematic review and meta analysis. J Am Heart Assoc. 2013;2(1):e004473.

8. Cornelissen VA, Fagard RH, Coeckelberghs E, Vanhees L. Impact of resistance training on blood pressure and other cardiovascular risk factors: a metaanalysis of randomized, controlled trials. Hypertension. 2011;58(5):950-8.

9. Pescatello LS, Franklin BA, Fagard R, Farquhar WB, Kelley GA, Ray CA. American College of Sports Medicine position stand: exercise and hypertension. Med Sci Sports Exerc. 2004;36(3):533-53.

10. Ruivo JA, Alcântara P. Hipertensão arterial e exercício físico [Hypertension and exercise]. Rev Port Cardiol. 2012;31(2):151-8. Portuguese

11. Franklin BA, Whaley $M H$, Howley ET, editors. ACSM's guidelines for exercise testing and prescription. 6th ed. Baltimore: Lippincott Williams \& Wilkins; 2000. ISBN 9780683303551

12. Kodama S, Saito K, Tanaka S, Maki M, Yachi Y, Asumi M, et al. Cardiorespiratory fitness as a quantitative predictor of all-cause mortality and cardiovascular events in healthy men and women: a meta-analysis. JAMA. 2009;301(19):2024-35.

\section{CONFLITO DE INTERESSES}

Os autores declaram não ter conflitos de interesses.

\section{ENDEREÇO PARA CORRESPONDÊNCIA}

José Pedro de Pinho Marques

Av. Hintze Ribeiro, $n^{\circ}$ 37, 3870-323 Torreira

E-mail: joseppmarques@gmail.com

Recebido em 01-09-2014

Aceite para publicação em 06-02-2015

\section{ABSTRACT}

\section{HYPERTENSION AND EXERCISE:THE BASIS FOR PRESCRIPTION}

Physical activity is associated with health benefits including a reduction in blood pressure (BP). A recent meta-analysis shows that exercise may reduce BP to a degree comparable to first-line BP-lowering drugs.

It was originally thought that the BP-lowering effect of exercise was limited to endurance exercise and that resistance or isometric exercise was harmful. Recent evidence contests this idea. The evidence is scarce but suggests a role for exercise in the primary prevention of hypertensive disease. The favourable influence of exercise on important risk factors such as hypertriglyceridemia and overweight has already been demonstrated.

Exercise is important in the primary and secondary prevention of hypertension. For most patients, the benefits of exercise largely exceed the risks. For individuals at risk, medical evaluation is indicated in order to allow safe and patient-oriented prescription of exercise.

Keywords: Hypertension, Exercise, Physical Activity. 